\title{
Why we should Boycott DEBRA
}

\author{
James Wheeler \\ Department of Radiation Oncology, Goshen Center for Cancer Care, Indiana
}

*Corresponding author: James Wheeler, Department of Radiation Oncology, Goshen Center for Cancer Care, Goshen, Indiana. Electronic

\section{Opinion}

Although this might mistakenly be seen as an issue for the radiation oncology community, all of us who share in the treatment of women with early stage breast cancer should be concerned about the upcoming NRG BR007 trial, a "Phase III Randomized Trial: Evaluating De-escalation of Breast Radiation (DEBRA) for Conservative Treatment of Stage I, HR+, HER2-, RS $\leq 18$ Breast Cancer), which is due to open in July. I am very supportive of the concept of using genomic testing to determine which patients between the ages of 50 and 70 with T1 N0 ER and/or PR positive breast cancers following breast conserving surgery with negative margins can be safely treated with endocrine therapy without adjuvant radiation therapy. Conceptually it is an exciting and well needed study. I am deeply concerned about the non-inferiority threshold of $4 \%$. This has never previously been considered to be an acceptable difference in ipsilateral breast tumor recurrence (IBTR) for women between the ages of 50 and 70 with stage I, low risk cancers.

The long term follow-up of NSABP B-39/RTOG 0413 (Lancet. 2019 December 14; 394(10215): 2155-2164. doi: 10.1016/S0140$6736(19) 32514-0)$ reported a $0.7 \%$ IBTR difference at 10 years (4.6\% in the accelerated partial breast irradiation (APBI) group versus $3.9 \%$ in the whole breast group). The ASTRO consensus guidelines (Practical Radiation Oncology. 2017; 7: 73-79. doi: 10.1016/j.prro.2016.09.007) currently place many additional restrictions on who is considered in the suitable, cautionary, or unsuitable categories despite the fact that none of these restrictions were placed on women treated in the B-39/0413 study. The B-39/0413 study was open to women at least 18 years of age with stage 0 , I, or II breast cancer with a unifocal primary $3 \mathrm{~cm}$ or less, pathology of either DCIS or invasive adenocarcinoma of the breast, negative margins (no tumor on ink), placed no restriction on ER or PR status, and allowed patients with 1-3 positive nodes provided there was a minimum of six resected axillary lymph nodes if the sentinel lymph node was positive. To date, I am unaware of any subgroup analysis of this trial demonstrating a greater than $1 \%$ difference in IBTR with APBI versus whole breast radiation for any of the patients now considered "cautionary" or even "unsuitable," including the node positive patients. The additional restrictions of margins negative by at least $3 \mathrm{~mm}$, size less than $2.5 \mathrm{~cm}$, age criteria, node negative, and ER status were all added to ensure that women who had accelerated partial breast radiation had even less than a $0.7 \%$ higher likelihood of IBTR with APBI than with whole breast radiation.

Intraoperative Radiation Therapy (IORT) is currently considered by the ASTRO consensus guidelines to be investigational, to be best performed within a clinical trial. The five year results of TARGITIORT (BMJ2020; 370:m2836. doi: 10.1136bmj.m2836) reported an IBTR of $2.11 \%$ with IORT compared to $0.95 \%$ with whole breast radiation, a difference of $1.16 \%$. This was accomplished with far broader criteria than the more restrictive ASTRO guidelines. TARGIT allowed women age 45 or greater compared to the ASTRO recommendation of age at least 50 years. TARGIT allowed a tumor size up to $3.5 \mathrm{~cm}$ compared to the ASTRO recommended upper limit of $2.5 \mathrm{~cm}$. TARGIT had no restriction on estrogen receptor (ER) status compared to the ASTRO restriction to ER positive patients. TARGIT allowed institutional preference regarding adding whole breast radiation for patients with positive axillary nodes, while ASTRO requires pN0. The margins for patients on the TARGIT trial were required to be negative by at least $1 \mathrm{~mm}$, compared to the ASTRO guidelines of a $3 \mathrm{~mm}$ negative margin. These guidelines remain in place because the ASTRO consensus panelists considered an increase in IBTR rate at 5 years of $1.2 \%$ to be excessively high.

The Early Breast Cancer Trialists Collaborative Group (EBCTCG) evaluated 42,000 women in 78 randomized trials. Their study demonstrated that approximately $75 \%$ of the local recurrences occurred within the first five years (Lancet 2005; 366: 20872106). Based on this observation, for those undergoing IORT, the difference in IBTR at ten years should only be $1.6 \%$ greater than for those receiving whole breast radiotherapy.

The concern regarding the dangers of an IBTR is well founded. The same EBCTCG study also highlighted the importance of reducing the risk of an ipsilateral breast recurrence. Only $75 \%$ of patients with IBTR are successfully salvaged. The remaining $25 \%$ have a significantly decreased 15 -year overall survival. To allow a $4 \%$ difference in IBTR for women using genomic profiling to avoid 
radiation therapy means that $1 \%$ of women will ultimately die of recurrent breast cancer whose death could have been prevented with the appropriate use of adjuvant radiation therapy. This has never previously been considered appropriate or acceptable for this group of women in their fifties and sixties with early stage, low risk breast cancers.

It is essential to insist on a non-inferiority limit no greater than $1.5 \%$. With this limit, less than $0.4 \%$ of women who do not receive adjuvant radiotherapy due to favorable genomic profiling will die as a long range consequence of an IBTR. This is probably a reasonable trade off to avoid the long term adverse effects of radiation therapy, particularly the cardiac effects in women with significant cardiac risk factors (active smokers, hypertension, obesity, diabetes, etc.).

The NRG has selected for this trial a group of women with low risk breast cancer for whom the estimated risk of IBTR in the radiotherapy group over ten years is $2.2 \%$. We have known for over 30 years from the classical studies such as NSABP B06 that for patients with early stage breast cancer, radiation therapy eliminates one-half to two-thirds of the local recurrences. Even without genomic profiling, the risk of recurrence in the observation group is only 4.4 to $6.6 \%$, resulting in a difference in IBTR of 2.2 to $4.4 \%$. Even without genomic profiling, the current $4 \%$ noninferiority threshold is likely to be met. Applying the BR007 trial IBTR estimates, we should insist on a non-inferiority limit no more than $1.5 \%$, expecting a 10 -year event-free survival of $97.8 \%$ for the radiotherapy group versus $96.3 \%$ for the omission of radiotherapy group. If genomic profiling cannot meet this standard, the trial should be considered a failure, and this form of genomic profiling should not be incorporated into clinical practice. To allow a $4 \%$ difference is a great disservice to women and would result in a large number of premature deaths that could have been avoided with radiation therapy.

There is great potential for genomic therapy. There is an understandable desire to avoid overtreatment of women with early stage breast cancer. We should, nonetheless, insist that the incorporation of genomic profiling meet the long term safety standards we demand of radiation therapy. Unless the noninferiority limit is placed no higher than $1.5 \%$, then all those who care for or about women with early stage breast cancer should boycott DEBRA.
This work is licensed under Creative Commons Attribution 4.0 License

To Submit Your Article Click Here: Submit Article
DOI: 10.32474/OAJOM.2021.04.000188

Citation: James Wheeler. Why we should Boycott DEBRA. Open Acc J Oncol Med 4(3)- 2021. OAJOM.MS.ID.000188. D0I: 10.32474/ OAJOM.2021.04.000188.

\section{OAJOM}

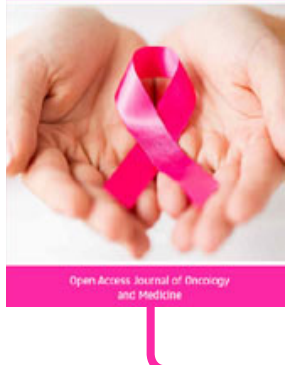

\section{Open Access Journal of Oncology} and Medicine

\section{Assets of Publishing with us}

- Global archiving of articles

- Immediate, unrestricted online access

- Rigorous Peer Review Process

- Authors Retain Copyrights

- Unique DOI for all articles 